\title{
Comparison of the determination of a low concentration active ingredient in pharmaceutical tablets by backscatter and transmission Raman spectrometry
}

\author{
Nichola Townshend, ${ }^{1}$ Alison Nordon, ${ }^{* 1}$ David Littlejohn, ${ }^{1}{ }^{1}$ Michael Myrick, ${ }^{2}$ John \\ Andrews $^{3}$ and Paul Dallin ${ }^{3}$ \\ ${ }^{1}$ WestCHEM, Department of Pure and Applied Chemistry and CPACT, University of \\ Strathclyde, 295 Cathedral Street, Glasgow, G1 1XL, UK \\ 2 Department of Chemistry and Biochemistry, University of South Carolina, Columbia, \\ SC 29208, USA \\ 3 Clairet Scientific Ltd., 17/18 Scirocco Close, Moulton Park Industrial Estate, \\ Northampton, NN3 6AP, UK \\ * denotes authors to whom correspondence should be sent \\ (Email: d.littlejohn@strath.ac.uk and alison.nordon@strath.ac.uk)
}

\begin{abstract}
383 tablets of a pharmaceutical product were analysed by backscatter and transmission Raman spectrometry to determine the concentration of an active pharmaceutical ingredient (API), chlorpheniramine maleate, at the $2 \% \mathrm{~m} / \mathrm{m}$ (4 mg) level. As the exact composition of the tablets was unknown, external calibration samples were prepared from chlorpheniramine maleate and microcrystalline cellulose (Avicel) of different particle size. The API peak at $1594 \mathrm{~cm}^{-1}$ in the $2^{\text {nd }}$ derivative Raman spectra was used to generate linear calibration models. The API concentration predicted using backscatter Raman measurements was relatively insensitive to the particle size of Avicel. With transmission, however, particle size effects were greater and accurate prediction of the API content was only possible when the photon propagation properties of the calibration and sample tablets were matched. Good agreement was obtained with HPLC analysis when matched calibration tablets were used for both modes. When the calibration and sample tablets are not chemically matched, spectral normalisation based
\end{abstract}


on calculation of relative intensities cannot be used to reduce the effects of differences in physical properties. The main conclusion is that although better for whole tablet analysis, transmission Raman is more sensitive to differences in the photon propagation properties of the calibration and sample tablets. 


\section{Introduction}

Analysis of tablets is an important part of quality control in pharmaceutical manufacturing. Near infrared spectrometry (NIRS) has proved useful for this assay, ${ }^{1}$ but in recent years there has been a growing interest in Raman spectrometry for tablet analysis owing to the greater chemical specificity, larger penetration depths into the sample and sharper spectral features compared to NIRS. Furthermore, common nonaromatic, non-crystalline excipients are generally poor Raman scattering materials in comparison to active drug substances that are often small aromatic heterocycles. Identifying active pharmaceutical ingredient (API) peaks, even if present in very low concentrations compared to the excipient, is therefore more likely to be achieved with Raman spectrometry, than with NIR spectrometry. ${ }^{2}$

Conventional backscatter Raman spectrometry, typically using laser beam diameters of $50-500 \mu \mathrm{m}$, has been used for the composition monitoring of pharmaceutical tablets. ${ }^{2-17}$ In many applications, advantages have been demonstrated over alternative techniques, often for determination of high relative concentrations of the analyte (up to 90\%). However, a number of authors have reported that the small volume of the tablet sampled by conventional Raman spectrometry can affect the accuracy and precision of the analysis. ${ }^{3,7,14}$ In a number of studies, a rotating sample holder ${ }^{6-7}, 10-11,13$, 15,17 or multiple-point irradiation ${ }^{7,14,16}$ was employed to increase the sampling area and hence, improve the repeatability of the Raman measurements. In an on-line example, the sampling area was increased by measuring a stream of tablets moving underneath the Raman probe on a conveyor system. ${ }^{17} \mathrm{Kim}$ et al. reported the advantages of wide area illumination for backscatter Raman analysis of pharmaceutical tablets. ${ }^{18}$ The study compared the performance of a Raman system that produced a $6 \mathrm{~mm}$ diameter spot size with that of a conventional Raman system with a $100 \mu \mathrm{m}$ spot size, to determine naproxen in tablets over the concentration range of $58-79 \% \mathrm{~m} / \mathrm{m}$. When replicate measurements were made on the same tablet using both methods, a significant improvement in the repeatability of the analyte peak area was seen with the larger spot size. Wide area illumination Raman analysis was also shown to be successful for the quantitative measurement of the API in an intact capsule formulation. ${ }^{19}$ 
Although an improvement over conventional optical configurations that employ a small laser spot size, wide area backscatter Raman spectrometry does not analyse the whole tablet. Matousek and Parker demonstrated that with a sample spot size of $4 \mathrm{~mm}$, $88 \%$ of the backscatter Raman signal from a $4 \mathrm{~mm}$ thick tablet was generated in the top $1 \mathrm{~mm}$ layer and only $12 \%$ of the signal originated from the remaining $3 \mathrm{~mm}$ of sample. ${ }^{20}$ Hence, backscatter Raman spectra of APIs can be dominated by Raman or fluorescence signals arising from the tablet coating or capsule. ${ }^{21-24}$ Research into Raman photon migration $^{25-26}$ and novel sampling configurations ${ }^{27-28}$ has revealed that many of the problems associated with backscatter Raman are removed when transmission Raman is used, and the attributes of the technique for analysis of pharmaceutical tablets and capsules are now being demonstrated. ${ }^{20,22,24,29-32}$ The absence of sub-sampling limitations in transmission Raman spectroscopy has been illustrated by numerical simulations ${ }^{20}$ and experimental investigations. ${ }^{20,33-36}$ Matousek and Parker showed that when a $0.5 \mathrm{~mm}$ thick inter-layer was moved from the sample surface to a depth of $3 \mathrm{~mm}$ within a tablet medium, the simulated backscatter Raman signal decreased by four orders of magnitude. ${ }^{20}$ In comparison, numerical ${ }^{20,34}$ and experimental ${ }^{34-36}$ investigations showed that the signal was only weakly dependent on the depth of the inter-layer in transmission mode; a reduction in signal was observed when the inter-layer was located at the sample surface owing to loss of laser and Raman photons at the air-sample interface. Elimination of the sub-sampling problem in transmission mode measurements has also been illustrated experimentally using two-layer tablets. ${ }^{20,}{ }^{33}$ While backscatter Raman spectra were dominated by signals from the material that was irradiated directly by the laser, transmission Raman spectra contained signals from the two materials for both tablet orientations.

There have been some reported applications of transmission Raman spectrometry for quantification of an API in pharmaceutical tablets. In most cases, the calibration and sample tablets had the same composition and physical properties, which simplified the analysis. Johansson et al. ${ }^{30}$ analysed 20 test tablets $(3.3 \mathrm{~mm}$ thick) that contained API concentrations between 16 and $24 \% \mathrm{~m} / \mathrm{m}$. Spectra were preprocessed using a standard normal variate (SNV) transformation to normalise the spectra and correct for baseline offsets. A laser excitation wavelength of $785 \mathrm{~nm}$ and a spot size of $6 \mathrm{~mm}$ in diameter 
were used and the study concluded that transmission Raman had the potential to give simpler more robust calibration models compared with backscatter Raman. This was primarily attributed to the more representative measurements achieved with transmission Raman, which resulted in a lower prediction error and the requirement for fewer partial least squares (PLS) components to explain the variance of the data. In a subsequent study, ${ }^{37}$ different multivariate methods were used to analyse transmission Raman data acquired of paracetamol tablets. Owing to the high spectral selectivity and bulk sampling properties of transmission Raman, it was possible to construct calibration models (PLS, multivariate curve resolution or classical least squares) using a reduced number of calibration samples as well as with fewer components. The simpler calibration requirements of transmission Raman spectrometry, compared to NIRS, offer considerable opportunities for deployment in drug development.

Eliasson et al. ${ }^{29}$ demonstrated the advantages of transmission Raman over the backscatter mode for the determination of $27-33 \% \mathrm{~m} / \mathrm{m}$ concentrations of an API contained within capsules, which gave rise to a strong interfering Raman signal (mainly $\mathrm{TiO}_{2}$ ). The signal from the capsule shell relative to that from the API was significantly reduced with transmission measurements and a root mean square error of prediction of $1.2 \%$ was achieved for the API with a $5 \mathrm{~s}$ acquisition time. Hargreaves et al. ${ }^{31}$ used transmission Raman measurements to quantify the concentration of API (approx. 75\% $\mathrm{m} / \mathrm{m}$ ) in a formulation containing three excipients in capsules of different sizes and fill level. Spectra were baseline corrected and normalised to unit length to account for variations in the transmission Raman signal intensity arising from differences in the amount of material within the capsules. Consequently, it was possible to use a PLS model developed using $100 \mathrm{mg}$ capsules to predict the API content in $100-400 \mathrm{mg}$ capsules with a relative error of less than $3 \%$.

Recently, transmission and backscatter Raman spectrometries were compared for quantification of two polymorphic forms (I and III) of flufenamic acid. ${ }^{38}$ Spectra were pre-processed using a multiplicative scatter correction. Measurements made in the backscatter mode were highly variable and over-estimated the amount of form III in mixtures of the two polymorphs. The high variability in the Raman signal was due to the inherent sub-sampling issue associated with backscatter measurements. Over-estimation 
of the amount of form III arose as a result of surface segregation of the two polymorphs and the bias of backscatter measurements towards the surface. In comparison, transmission measurements were not affected by any sub-sampling issues and so provided an accurate method for quantification of the two polymorphs.

The aforementioned studies indicate that Raman spectrometry, particularly in the transmission mode, is well suited for the quantitative, non-invasive analysis of pharmaceutical tablets. Most of the reported applications have concerned determination of API concentrations in excess of $10 \% \mathrm{~m} / \mathrm{m}$ and the number of tablets analysed has generally been 20 or less, and often involved simulated samples. In this study, a large number (383) of tablets of a commercially available pharmaceutical product has been analysed by backscatter and transmission Raman spectrometry to determine the concentration of an API at the $2 \% \mathrm{~m} / \mathrm{m}$ level. In previous studies comparing the performance of backscatter and transmission Raman spectrometries, the calibration and sample tablets were generally matched. However, in one study ${ }^{31}$ where the amount of material analysed was varied but the chemical composition of the calibration and sample tablets was matched, it was possible to use spectral normalisation to unit length to render the transmission measurement insensitive to the amount of material. When the full composition of the samples is unknown, it is not possible to use matched calibration standards. In this case, the aforementioned normalisation procedure based on calculation of relative intensities cannot be used to correct for possible differences in the photon propagation properties of the calibration and sample tablets. In the present study, external calibration tablets that were not matched to the composition of the sample tablets were prepared from different particle size distributions of microcrystalline cellulose and chlorpheniramine maleate, which produced calibration tablets with different photon propagation properties. The susceptibility of backscatter and transmission Raman analysis to such variations in the physical properties of sample and calibration tablets, which may arise when external calibration tablets are used, was compared. This type of assessment has not been previously reported. 


\section{Experimental}

\section{Materials}

Chlortrimeton antihistamine over-the-counter tablets (Schering-Plough HealthCare Products, Inc., Memphis, TN, USA) were purchased from a pharmacy. The tablets came from the same manufactured batch and nominally contained $4 \mathrm{mg}$ (about $2 \% \mathrm{~m} / \mathrm{m}$ ) chlorpheniramine maleate (the API), corn starch, D\&C yellow No $6 \& 10$, lactose monohydrate and magnesium stearate, although the exact composition of the samples was unknown.

Chlorpheniramine maleate (99.8\% purity), reagent grade ammonium acetate, HPLC grade acetonitrile and reagent grade glacial acetic acid were purchased from Sigma Aldrich, USA. Microcrystalline cellulose (Avicel PH-101, 105 and 200) was provided by GSK, Irvine, UK. The particle size information $\left(D_{10}-D_{90}\right.$ and $\left.D_{50}\right)$ for each grade of Avicel has been reported as: ${ }^{39} \mathrm{PH}-101: 20.5-140.9 \mu \mathrm{m}$ and $61.7 \mu \mathrm{m}$; PH-105: $4.5-36.0 \mu \mathrm{m}$ and $16.3 \mu \mathrm{m}$; PH-200: $41.1-333.8 \mu \mathrm{m}$ and $178.0 \mu \mathrm{m}$.

\section{High performance liquid chromatography (HPLC)}

A Waters 660 pump with a 626S controller, in-line degasser, 717 autosampler and diode array detector were used. Data were acquired and processed using Waters Empower software. Separation was achieved with an Agilent column (C18, $4.6 \mathrm{~mm} \times$ $150 \mathrm{~mm}, 5 \mu \mathrm{m}$ particle size $)$ operated at ambient temperature $\left(20-23{ }^{\circ} \mathrm{C}\right)$. The mobile phase was an 80:20 mix of $25 \mathrm{mM}$ ammonium acetate and acetonitrile, adjusted to $\mathrm{pH} 5.5$ using glacial acetic acid.

Seven solutions of chlorpheniramine maleate (covering the range $0-240 \mathrm{mg} \mathrm{L}^{-1}$ ) were prepared in the mobile phase for calibration of the instrument response. Tablets were crushed and the powder dissolved in mobile phase and diluted to $25 \mathrm{~mL}$. As the intended mass of chlorpheniramine maleate in each tablet is $4 \mathrm{mg}$, the concentration of chlorpheniramine maleate in the sample solutions was about $160 \mathrm{mg} \mathrm{L}^{-1}$. Solutions were sonicated for five minutes and then filtered using $0.2 \mu \mathrm{m}$ disposable Acrodisc filters (Sigma Aldrich, USA) before injecting $20 \mu \mathrm{L}$ for analysis. The chromatogram recorded at 
$254 \mathrm{~nm}$ revealed the analyte peak at $1.71 \mathrm{~min}$ and two excipient peaks at 10 and $12.5 \mathrm{~min}$. The relative standard deviation (RSD) of the analyte peak areas for six replicate injections of a $160 \mathrm{mg} \mathrm{L}^{-1}$ chlorpheniramine maleate standard solution was $0.93 \%$. The calibration response curve for chlorpheniramine maleate was linear over the range 0 $240 \mathrm{mg} \mathrm{L}^{-1}\left(\mathrm{R}^{2}=0.9986\right)$.

\section{Raman spectrometry}

A Kaiser Raman Workstation and PhAT probe (Kaiser Optical systems, Ann Arbor, USA) were used for tablet analysis in the backscatter and transmission modes. An Invictus diode laser was employed, which has a wavelength of $785 \mathrm{~nm}$ and was operated at $400 \mathrm{~mW}$ at the source. For backscatter measurements, the tablets were illuminated from above using the PhAT probe with a laser beam optically expanded to give a $3 \mathrm{~mm}$ spot size. For transmission measurements, the tablets were illuminated from below using a laser spot that had been expanded to $3 \mathrm{~mm}$ diameter and transmitted Raman photons were collected from a $3 \mathrm{~mm}$ diameter circular region on the upper surface of the tablet directly above the illumination point. Raman spectra were obtained with a $20 \mathrm{~s}$ exposure time and 3 acquisitions for backscatter measurements, and a $60 \mathrm{~s}$ exposure time and 3 acquisitions for transmission measurements. A dark current spectrum was acquired before each set of experiments and the HoloGRAMS operating software subtracted the dark current from each sample spectrum acquired. The spectra were then converted to text files and imported into Matlab version 7.0.2 (Mathworks Inc., Natick, Massachusetts, USA) for analysis using the PLS_Toolbox version 3.0.4 (Eigenvector Research Inc., Manson, Washington, USA). Second derivative spectra were calculated using the Savitzky-Golay function with a 51 point filter width and second order polynomial.

Calibration tablets containing known concentrations of chlorpheniramine maleate were prepared for direct analysis of the Chlortrimeton tablets. As the detailed composition of the sample tablets could not be obtained from the manufacturer, microcrystalline cellulose (Avicel) was used to produce the calibration tablets as it did not interfere with the spectral region of interest (Figure 1). Avicel and chlorpheniramine maleate were blended for $10 \mathrm{~min}$ in a tumbler blender and then tablets were pressed for 


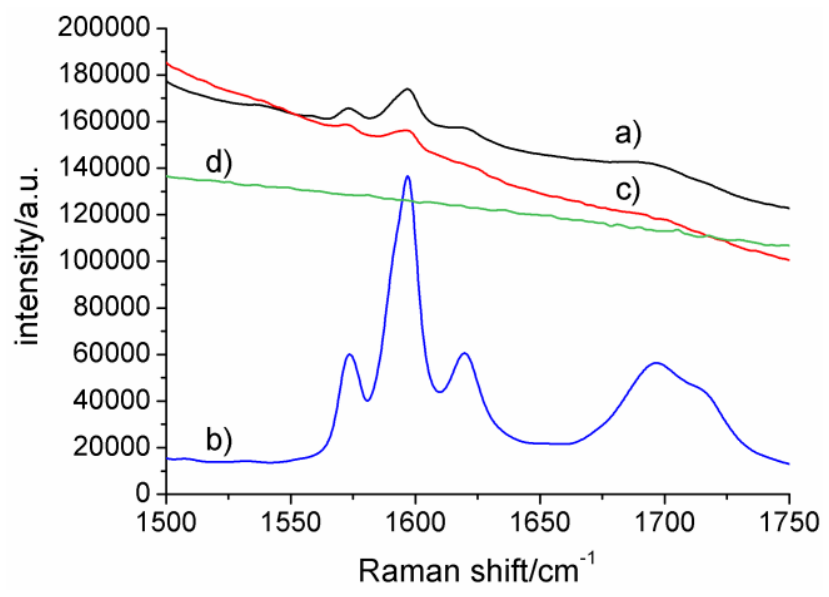

Figure 1. Raman spectra of a) a Chlortrimeton tablet (backscatter), b) a powdered sample of chlorpheniramine maleate (backscatter), c) a Chlortrimeton tablet (transmission, signal multiplied by 6) and d) a sample of Avicel $\mathrm{PH}-101$ powder (transmission, signal multiplied by 6 ). 
each analyte concentration. The calibration tablets were weighed to estimate the mass of chlorpheniramine maleate in each tablet, assuming that a homogeneous blend had been produced. Six tablets covering the range $0-8 \mathrm{mg}$ of chlorpheniramine maleate per tablet were used for analysis of 383 Chlortrimeton tablets by backscatter and transmission Raman measurements.

Calibration plots were produced using the intensity of the chlorpheniramine maleate peak at $1594 \mathrm{~cm}^{-1}$, from both sides of each tablet, scaled to account for fluctuations in laser power. The scaling factor for each spectrum was calculated from the intensity of the Avicel peak at $1095.5 \mathrm{~cm}^{-1}$ in the $2^{\text {nd }}$ derivative spectrum of the first calibration tablet analysed divided by the Avicel peak intensity in the spectrum of subsequent calibration tablets. All the calibration plots had $\mathrm{R}^{2}$ values between 0.98 and 0.99. Similarly, the intensity of the chlorpheniramine maleate peak in the Chlortrimeton tablets was scaled using the lactose ${ }^{40}$ excipient peak at $1142.5 \mathrm{~cm}^{-1}$ in the $2^{\text {nd }}$ derivative spectrum of the first tablet analysed divided by the lactose peak intensity in the spectrum of subsequent sample tablets. The scaled peak intensities were then used to calculate the mass of chlorpheniramine maleate in the Chlortrimeton tablets. 


\section{Results}

\section{HPLC analysis}

254 tablets were individually weighed and analysed to determine the mass of chlorpheniramine maleate in each tablet. A summary of the results is given in Table 1 and histograms of tablet mass and amount of chlorpheniramine maleate are shown in Figures $2 \mathrm{a}$ and $2 \mathrm{~b}$, respectively. The mean amount of $3.98 \mathrm{mg}$ chlorpheniramine maleate was close to the target value of $4 \mathrm{mg}$ indicated by the manufacturer, but the range was quite wide (3.36 to $4.81 \mathrm{mg}$ ) and the mass of the tablets also varied more than expected. The tablet mass and amount of chlorpheniramine maleate did not always vary proportionally; some tablets of higher mass had lower API content and vice versa, such that the chlorpheniramine maleate concentration ranged from 1.66 to $3.31 \% \mathrm{~m} / \mathrm{m}$ (Figure $2 \mathrm{c}$ ). Skewness and kurtosis statistics show that the data for the tablet mass, and API mass and concentration are not normally distributed. The API data are skewed to the right and are long tailed, which means that there is an enhanced probability of a tablet containing a high API content compared to a normal distribution. As the Chlortrimeton tablets are intended to be taken four times daily for a prolonged period, the variation in the amount of chlorpheniramine maleate contained in the tablets is of no pharmaceutical consequence. The results suggest, however, that better control of the manufacturing process could be achieved and in this regard direct spectroscopic analysis at the blending and tableting steps has potential advantages over HPLC, which is destructive and time consuming.

\section{Raman spectrometry}

Spectra of both sides of a tablet with heterogeneous distribution of chlorpheniramine maleate. A calibration tablet was prepared with a heterogeneous distribution of chlorpheniramine maleate $(6.59 \mathrm{mg}$ in a mass of Avicel corresponding to $4.95 \% \mathrm{~m} / \mathrm{m}$ analyte) and both sides of the tablet were analysed by backscatter and transmission Raman spectrometry. The spectra in Figure 3a show that in the backscatter mode, the heterogeneous distribution of chlorpheniramine maleate results in spectra of different intensity from the two sides of the tablet. In contrast, the transmission mode spectra 
Table 1. Summary of maximum, minimum, mean, \%RSD, skewness and kurtosis values of the tablet mass, and chlorpheniramine maleate mass and concentration for 254 Chlortrimeton tablets.

\begin{tabular}{|c|c|c|c|}
\hline & tablet mass & $\begin{array}{c}\text { Chlorpheniramine maleate } \\
\text { mass }\end{array}$ & $\begin{array}{c}\text { Chlorpheniramine maleate } \\
\text { concentration }\end{array}$ \\
\hline Mean & $201.4 \mathrm{mg}$ & $3.98 \mathrm{mg}$ & $1.98 \% \mathrm{~m} / \mathrm{m}$ \\
\hline Minimum & $118.5 \mathrm{mg}$ & $3.36 \mathrm{mg}$ & $1.57 \% \mathrm{~m} / \mathrm{m}$ \\
\hline Maximum & $250.7 \mathrm{mg}$ & $4.81 \mathrm{mg}$ & $3.31 \% \mathrm{~m} / \mathrm{m}$ \\
\hline$\%$ RSD & 6.1 & 6.3 & 8.8 \\
\hline Skewness $^{\mathrm{a}}$ & -0.91 & 1.06 & 1.99 \\
\hline Kurtosis $^{\mathrm{a}}$ & 12.43 & 5.01 & 15.45 \\
\hline
\end{tabular}

${ }^{a}$ Skewness and kurtosis statistics were calculated using the third and fourth standardised moments, respectively. ${ }^{41}$ 

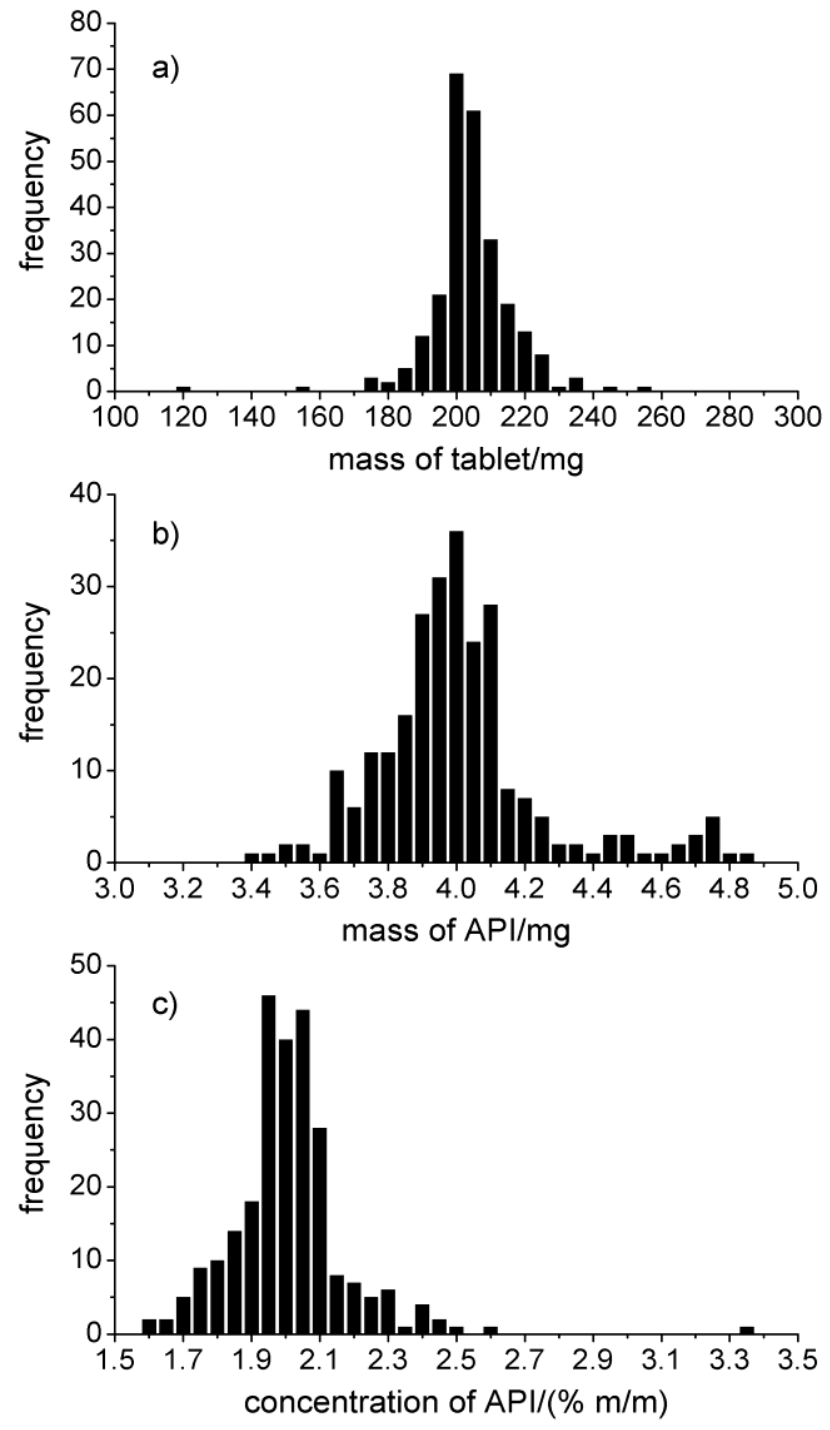

Figure 2. Histograms of a) tablet mass, b) chlorpheniramine maleate (API) mass and c) chlorpheniramine maleate (API) concentration $(\mathrm{n}=254)$. 

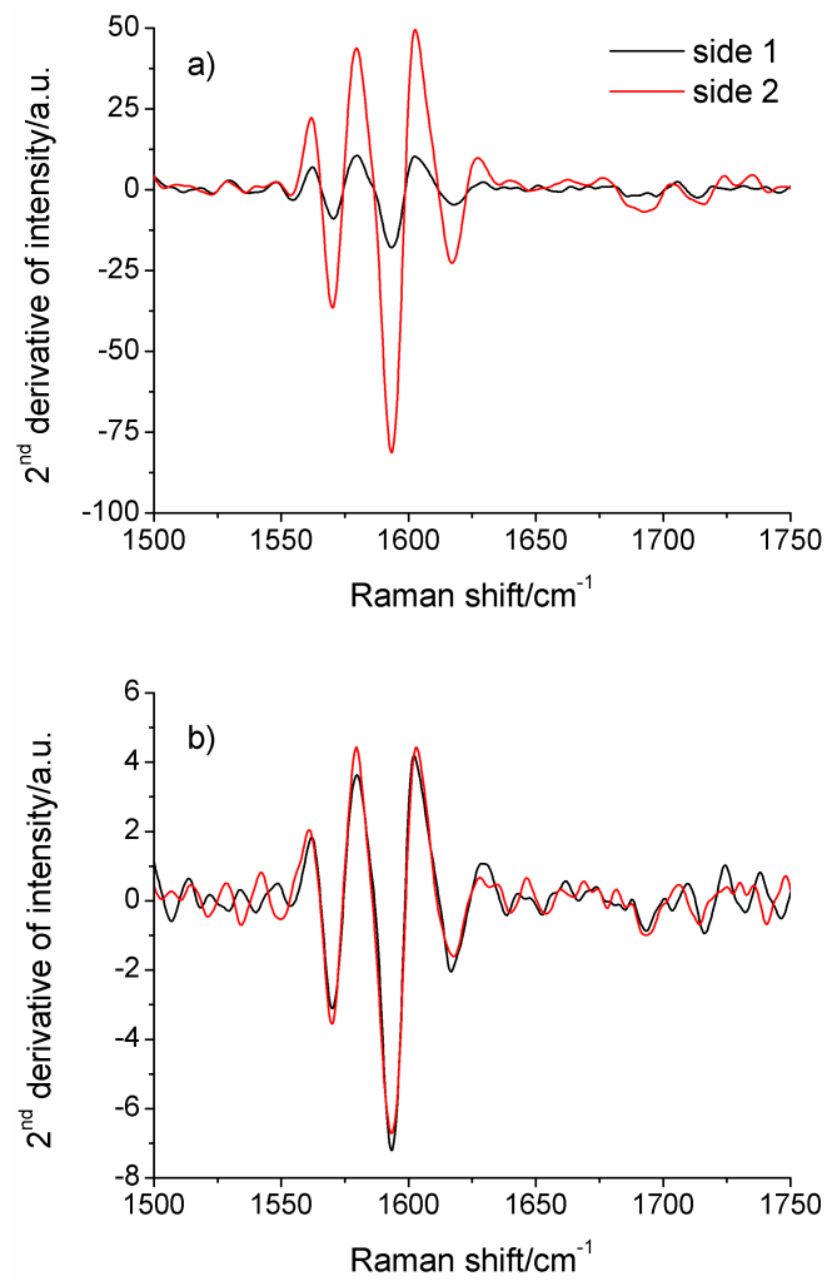

Figure 3. Second derivative Raman spectra of both sides of a microcrystalline cellulose based tablet (Avicel PH-101) showing the chlorpheniramine maleate peak at $1594 \mathrm{~cm}^{-1}$ in the a) backscatter and b) transmission modes. 
(Figure 3b) are more similar in intensity. Backscatter Raman maps acquired of both sides of the tablet using the PhAT probe showed that one surface of the tablet was rich in chlorpheniramine maleate whereas the other side was predominantly excipient. Hence, the results obtained are similar to those reported for the analysis of two-layer tablets using backscatter and transmission Raman measurements. ${ }^{20,33}$ It can be concluded that backscatter Raman is better at indicating differences in the amount of a compound on different sides of a tablet (i.e. heterogeneity), whereas transmission Raman should be better for whole tablet quantitative analysis.

Analysis of Chlortrimeton tablets using external calibration tablets. A second set of 383 Chlortrimeton tablets were analysed by backscatter and transmission Raman spectrometry using calibration tablets prepared with Avicel PH-101 microcrystalline cellulose $\left(D_{50}\right.$ of $\left.61.7 \mu \mathrm{m}^{39}\right)$. The scaled intensity at $1594 \mathrm{~cm}^{-1}$ in the $2^{\text {nd }}$ derivative spectrum from both sides of each calibration tablet was used to generate the linear calibration models. When the sample tablets were analysed by backscatter Raman spectrometry, the mean mass of chlorpheniramine maleate was $3.87 \mathrm{mg}$ with a RSD of $3.3 \%$, in good agreement with the results obtained by HPLC for the first set of 254 Chlortrimeton tablets. However, with transmission Raman spectrometry the mean mass derived from the calibration model was $8.70 \mathrm{mg}$ with a RSD of 5.4\%, which suggests that there is a difference in the propagation of the Raman photons through the sample and calibration tablets caused by their different composition.

To investigate the effect of particle size on the backscatter and transmission Raman signals of chlorpheniramine maleate, additional calibration tablets were prepared from Avicel PH-105 and Avicel PH-200 that have $\mathrm{D}_{50}$ values of 16.3 and $178.0 \mu \mathrm{m}$, respectively. ${ }^{39}$ The average mass of the API predicted by backscatter and transmission Raman spectrometry using the calibration tablets prepared from each grade of Avicel are shown in Table 2. The effect of the particle size of Avicel on the accuracy of the estimated mass of chlorpheniramine maleate in the Chlortrimeton tablets is much greater for the transmission Raman measurements than for backscatter Raman. The calibration tablets made using Avicel PH-200 gave the most accurate prediction of chlorpheniramine maleate by transmission Raman suggesting that the photon transmission properties of 
Table 2. Comparison of the average predicted mass of chlorpheniramine maleate and $\%$ RSD values obtained for backscatter and transmission Raman measurements at $1594 \mathrm{~cm}^{-1}$ in $2^{\text {nd }}$ derivative spectra when calibration tablets containing different Avicel grades are used; $\mathrm{n}=383$; expected mass $4 \mathrm{mg}$.

\begin{tabular}{|c|c|c|c|c|}
\hline $\begin{array}{c}\text { Grade of } \\
\text { Avicel }\end{array}$ & \multicolumn{2}{|c|}{ Backscatter Raman } & \multicolumn{2}{c|}{ Transmission Raman } \\
\hline & Average mass/mg & \%RSD & Average mass/mg & \%RSD \\
\hline PH-105 & 4.32 & 3.5 & 7.33 & 5.6 \\
\hline PH-101 & 3.87 & 3.3 & 8.70 & 5.4 \\
\hline PH-200 & 3.64 & 3.6 & 3.95 & 5.8 \\
\hline
\end{tabular}


these tablets match best those of the Chlortrimeton tablets. This does not necessarily mean that the sample and Avicel PH-200 have similar particle sizes, only similar photon transmission efficiency, which may arise for a number of reasons. Although the Avicel particle size effects on the backscatter Raman measurements are less pronounced than for transmission Raman, there are some differences with the PH-101 grade giving the best estimate of the chlorpheniramine maleate content of the Chlortrimeton tablets.

The relative insensitivity of wide area backscatter measurements to differences in particle size between the calibration and sample tablets is consistent with previous findings. ${ }^{42} \mathrm{Hu}$ et al. attributed the insensitivity of wide area $(3 \mathrm{~mm}$ laser spot size) backscatter Raman measurements, compared to measurements made using a $60 \mu \mathrm{m}$ laser spot size, to the increased sampling volume being much greater than the sample particle size. ${ }^{42}$ Although the sampling volume of transmission Raman measurements far exceeds that of backscatter Raman measurements, which are dominated by the initial $1 \mathrm{~mm}$ or so of a tablet, ${ }^{20}$ previous studies have shown that the propagation of photons in the transmission mode is significantly affected by variations in the size and type of particles. $^{36,}{ }^{43}$ The relatively minor effect of particle size on the backscatter Raman spectra arises because most of the signal originates from the surface. ${ }^{20}$ Particle size affects the surface smoothness and it may be that this is best matched to the sample tablets in this study when the calibration tablets are prepared from Avicel PH-101.

In previous studies where the chemical composition and the physical properties of the sample and calibration tablets were matched, any effects of variations in the amount of material measured on the transmission Raman signal were reduced through normalisation procedures based on calculation of relative intensities. Although it is generally best to matrix-match the sample and calibration tablets, this is not always possible, especially if the sample tablet composition is not known accurately. This part of the study demonstrated that although transmission Raman measurements are more representative of the whole sample, in contrast to backscatter, it is more likely that inaccurate estimations of the analyte mass/concentration will arise if the external calibration set is not well matched to the sample set in terms of photon propagation characteristics. This is a conclusion that has not been mentioned in previous studies of 
transmission Raman spectrometry. Addition of an internal standard can mitigate against matrix differences between samples and calibrants, but is generally destructive ${ }^{10}$ and not suitable for direct analysis of tablets.

\section{Comparison of Raman and HPLC analyses}

A sub-set of 25 Chlortrimeton tablets analysed by both Raman modes was also analysed using the HPLC method to allow a direct comparison of the performance of the techniques on the same sample set. The results for backscatter and transmission Raman were obtained with the PH-101 and PH-200 Avicel calibration tablets, respectively. The mean mass of chlorpheniramine maleate \pm 1 standard deviation obtained by HPLC, backscatter and transmission Raman, respectively, was $3.85 \pm 0.11,3.92 \pm 0.20$ and $4.02 \pm 0.24 \mathrm{mg}$. Through the use of the F-test and Student's t-test, it was shown that there were no statistical differences at the $95 \%$ confidence interval between the average values obtained by the two Raman methods and between backscatter Raman and HPLC, but there were statistical differences between the results obtained by transmission Raman and HPLC. 


\section{Conclusions}

Previous work by the authors has shown that the physical properties of a powder (e.g. particle size) have a significant effect on the propagation of laser and Raman photons generated in the transmission mode. ${ }^{36}$ The results of the present investigation illustrate the analytical consequences of these prior observations for the analysis of tablets. It has been shown that transmission Raman spectrometry can be used to quantify accurately the content of chlorpheniramine maleate at the $2 \% \mathrm{~m} / \mathrm{m}$ level in a pharmaceutical tablet product when external calibration tablets are prepared from microcrystalline cellulose (Avicel), provided the photon propagation characteristics of the sample and calibration tables are matched. Backscatter Raman can also be used successfully for this analysis and is less susceptible to particle size effects. In previous Raman studies, the effects of physical properties such as particle size were either not explored or considered to be less significant than for near infrared spectrometry. ${ }^{32}$ This study indicates that this is not the case. Nonetheless, both Raman methods have advantages over HPLC which is often the standard method for API analysis, but it takes longer and involves destruction of the sample tablets.

If preparation of calibration tablets with photon propagation properties similar to the samples is not easily achieved, a reference method such as HPLC could be used to construct calibration models for the Raman analysis. However, it is likely that the API would need to be added to a selection of sample tablets to extend the calibration range as the mass range of API in commercial samples is normally relatively small. Recently there have been some advances in spectral pre-processing procedures for the removal of the effects of particle size from Raman spectra acquired in backscatter mode. ${ }^{44}$ Such procedures could be extended to provide similar benefits in transmission Raman spectrometry, particularly when it is not possible to use calibration and sample tablets with the same chemical composition. 


\section{Acknowledgements}

NT was supported by the Engineering and Physical Sciences Research Council and the Centre for Process Analytics and Control Technology (CPACT). The Royal Society is thanked for the award of a University Research Fellowship to AN. 


\section{References}

1. Kirsch, J. D.; Drennen, J. K. Appl. Spectrosc. Rev. 1995, 30, 139-174.

2. $\quad$ Mazurek, S.; Szostak, R. J. Pharm. Biomed. Anal. 2008, 48, 814-821.

3. Dyrby, M.; Engelsen, S. B.; Nørgaard, L.; Bruhn, M.; Lundsberg-Nielsen, L. Appl. Spectrosc. 2002, 56, 579-585.

4. Breitenbach, J.; Schrof, W.; Neumann, J. Pharm. Res. 1999, 16, 1109-1113.

5. Williams, A. C.; Cooper, V. B.; Thomas, L.; Griffith, L. J.; Petts, C. R.; Booth, S. W. Int. J. Pharm. 2004, 275, 29-39.

6. Wang, C.; Vickers, T. J.; Mann, C. K. J. Pharm. Biomed. Anal. 1997, 16, 87-94.

7. Johansson, J.; Pettersson, S.; Folestad, S. J. Pharm. Biomed. Anal. 2005, 39, 510516.

8. Bell, S. E. J.; Burns, D. T.; Dennis, A. C.; Matchett, L. J.; Speers, J. S. Analyst 2000, 125, 1811-1815.

9. Hausman, D. S.; Cambron, R. T.; Sakr, A. Int. J. Pharm. 2005, 299, 19-33.

10. Szostak, R.; Mazurek, S. Analyst 2002, 127, 144-148.

11. Szostak, R.; Mazurek, S. J. Mol. Struct. 2004, 704, 229-233.

12. Skoulika, S. G.; Georgiou, C. A. Appl. Spectrosc. 2001, 55, 1259-1265.

13. Mazurek, S.; Szostak, R. J. Pharm. Biomed. Anal. 2006, 40, 1225-1230.

14. Xie, Y.; Tao, W.; Morrison, H.; Chiu, R.; Jona, J.; Fang, J.; Cauchon, N. Int. J. Pharm. 2008, 362, 29-36.

15. Orkoula, M. G.; Kontoyannis, C. G. J. Pharm. Biomed. Anal. 2008, 47, 631-635.

16. Vergote, G. J.; Vervaet, C.; Remon, J. P.; Haemers, T.; Verpoort, F. Eur. J. Pharm. Sci. 2002, 16, 63-67. 
17. Wikström, H.; Romero-Torres, S.; Wongweragiat, S.; Williams, J. A. S.; Grant, E. R.; Taylor, L. S. Appl. Spectrosc. 2006, 60, 672-681.

18. Kim, M.; Chung, H.; Woo, Y.; Kemper, M. Anal. Chim. Acta 2006, 579, 209-216.

19. Kim, J.; Noh, J.; Chung, H.; Woo, Y.-A.; Kemper, M. S.; Lee, Y. Anal. Chim. Acta 2007, 598, 280-285.

20. Matousek, P.; Parker, A. W. Appl. Spectrosc. 2006, 60, 1353-1357.

21. Eliasson, C.; Matousek, P. Anal. Chem. 2007, 79, 1696-1701.

22. Macleod, N. A.; Matousek, P. Pharm. Res. 2008, 25, 2205-2215.

23. Macleod, N. A.; Matousek, P. Appl. Spectrosc. 2008, 11, 291A-304A.

24. Matousek, P.; Parker, A. W. J. Raman Spectrosc. 2007, 38, 563-567.

25. Everall, N.; Hahn, T.; Matousek, P.; Parker, A. W.; Towrie, M. Appl. Spectrosc. 2001, 55, 1701-1708.

26. Everall, N.; Hahn, T.; Matousek, P.; Parker, A. W.; Towrie, M. Appl. Spectrosc. 2004, 58, 591-597.

27. Matousek, P.; Clark, I. P.; Draper, E. R. C.; Morris, M. D.; Goodship, A. E.; Everall, N.; Towrie, M.; Finney, W. F.; Parker, A. W. Appl. Spectrosc. 2005, 59, $393-400$.

28. Matousek, P.; Everall, N.; Towrie, M.; Parker, A. W. Appl. Spectrosc. 2005, 59, 200-205.

29. Eliasson, C.; Macleod, N. A.; Jayes, L. C.; Clarke, F. C.; Hammond, S. V.; Smith, M. R.; Matousek, P. J. Pharm. Biomed. Anal. 2008, 47, 221-229.

30. Johansson, J.; Sparén, A.; Svensson, O.; Folestad, S.; Claybourn, M. Appl. Spectrosc. 2007, 61, 1211-1218. 
31. Hargreaves, M. D.; Macleod, N. A.; Smith, M. R.; Andrews, D.; Hammond, S. V.; Matousek, P. J. Pharm. Biomed. Anal. 2011, 54, 463-468.

32. Buckley, K.; Matousek, P. J. Pharm. Biomed. Anal. 2011, 55, 645-652.

33. Sparén, A.; Johansson, J.; Svensson, O.; Folestad, S.; Claybourn, M. Am. Pharm. Rev. 2009, 12, 62-71.

34. Matousek, P.; Everall, N.; Littlejohn, D.; Nordon, A.; Bloomfield, M. Appl. Spectrosc. 2011, 65, 724-733.

35. Everall, N.; Priestnall, I.; Dallin, P.; Andrews, J.; Lewis, I.; Davis, K.; Owen, H.; George, M. W. Appl. Spectrosc. 2010, 64, 476-484.

36. Townshend, N.; Nordon, A.; Littlejohn, D.; Andrews, J.; Dallin, P. submitted to Anal. Chem. 2011.

37. Fransson, M.; Johansson, J.; Sparén, A.; Svensson, O. J. Chemom. 2010, 24, 674680.

38. Aina, A.; Hargreaves, M. D.; Matousek, P.; Burley, J. C. Analyst 2010, 135, 2328-2333.

39. Narayan, P.; Hancock, B. C. Mater. Sci. Eng., A 2005, 407, 226-233.

40. de Veij, M.; Vandenabeele, P.; De Beer, T.; Remon, J. P.; Moens, L. J. Raman Spectrosc. 2009, 40, 297-307.

41. Thode, H. C., Testing for Normality. Marcel Dekker, Inc.: New York, 2002; Vol. 164.

42. Hu, Y.; Wikström, H.; Byrn, S. R.; Taylor, L. S. Appl. Spectrosc. 2006, 60, 977984.

43. Schrader, B.; Bergmann, G. Z. Anal. Chem. Fresenius 1967, 225, 230-247. 
44. Chen, Z.-P.; Li, L.-M.; Jin, J.-W.; Nordon, A.; Littlejohn, D.; Yang, J.; Zhang, J.; Yu, R.-Q. Anal. Chem. 2012, doi: 10.1021/ac300189p. 
for TOC only

$$
\left.\lambda_{\text {LASER }}\right\rfloor / \lambda_{\text {RAMAN }} \underset{\text { VS }}{\mid \lambda_{\text {LASER }}} \stackrel{\mid \lambda_{\text {RAMAN }}}{\longrightarrow} \text { API mass }
$$

